REVISTA CHILENA DE LITERATURA

Diciembre 2016, Número 94, 59-77

\title{
LA LECTURA EN FORMATOS DIGITALES EN EL CHILE ACTUAL: NUEVAS PRÁCTICAS Y VIEJAS DESIGUALDADES ${ }^{1}$
}

\author{
Cristóbal Moya \\ Universidad Diego Portales \\ cristobalmoya@gmail.com \\ Mónica M. Gerber \\ Universidad Diego Portales \\ monica.gerber@mail.udp.cl
}

\section{RESUMEN / ABSTRACT}

En el presente artículo se caracteriza la fisionomía de la lectura en formatos digitales, especialmente en comparación con los impresos. Para esto se analiza la lectura en formatos digitales de cuatro materiales de lectura en el Chile actual (libros, revistas, periódicos e historietas) a partir del Estudio de Comportamiento Lector 2014. Las prácticas lectoras son analizadas en cuanto prácticas culturales más amplias, lo que permite dar cuenta de la afinidad existente entre prácticas lectoras y determinados grupos sociales. En particular, se examinan las implicancias de ser un "lector omnívoro" en términos de formatos digitales e impresos. Se encuentra que los "lectores omnívoros" corresponden a perfiles lectores de posiciones sociales aventajadas en términos de educación e ingresos del hogar. Además, los omnívoros son quienes exhiben una mayor disposición a leer distintos materiales y en distintos formatos. Finalmente, se evidencia que existe una reproducción intergeneracional de la desigual disposición a leer en formatos digitales a partir del análisis de la educación de los padres.

Palabras clave: lectura digital en Chile, prácticas lectoras en Chile, lectura y distinción social, omnivorismo lector.

1 Este artículo se enmarca en el proyecto "Disposiciones a la lectura en Chile", folio 72149 , financiado por el Fondo del Libro, línea Investigación del Consejo Nacional del Libro y la Lectura, año 2015. Este trabajo también cuenta con apoyo del Centro de Estudios de Conflicto y Cohesión Social, CONICYT/FONDAP/15130009 COES. 
In this article the main features of the format of digital reading are presented, particularly in comparison with print. In order to carry out this aim, the reading in digital format of four reading materials in contemporary Chile (books, magazines, newspapers and comics) is analyzed drawing on the Estudio de Comportamiento Lector 2014 (Study of Reading Behavior). Reading practices are analyzed as broader cultural practices, which allows to establish links between reading practices and certain social groups. Particularly, the implications of being an 'omnivore reader' are examined, considering digital and print formats. Results show that 'omnivore readers' correspond to reading profiles of advantaged social positions in terms of education and household income. Furthermore, omnivores express a greater willingness to read different materials and formats. Finally, an intergenerational reproduction of the unequal disposition to reading in digital formats is evidenced when analyzing the parents reading education.

KEYWORDS: digital reading in Chile, reading practices in Chile, reading and social distinction, cultural omnivorousness.

\section{INTRODUCCIÓN}

El Chile actual puede considerarse como un país lector, en tanto prácticamente toda su población se encuentra alfabetizada ${ }^{2}$. Hace mucho tiempo ya que saber leer es demandado en la vida social y se constituye como una de las competencias básicas que se espera transmita el sistema educacional. No obstante lo extendido de la alfabetización, las lecturas y las formas de leer difieren sustantivamente. Los lectores se diferencian tanto por los formatos y materiales que leen, la frecuencia y la forma en que lo hacen, como por las valoraciones que tienen sobre la lectura y la literatura. Las prácticas lectoras difieren, además, entre distintos grupos sociales: existen variaciones importantes según el nivel educacional y el ingreso de las personas y sus padres (Cociña), lo que sugiere que los hábitos de lectura se relacionan con prácticas culturales más amplias y que se constituyen como una forma específica de distinción de determinados grupos sociales (Bourdieu). Tomando en consideración la transformación de los materiales de lectura impresos al transponerse en formato digital, así como la falta de atención a este fenómeno en Chile, resulta particularmente relevante examinar las particularidades de

2 La Unesco (2016) estima que para el año 2015, el 97,3\% de las personas de 15 o más años en Chile se cuentan como alfabetizadas. Este indicador se refiere al nivel más elemental de alfabetización, que considera la habilidad de leer, escribir y comprender un enunciado simple referido a la vida cotidiana. 
la lectura digital (Noyes y Garland) para evaluar si las desigualdades sociales en las prácticas lectoras en formato digital se condicen con las distinciones conocidas en impresos. Con este objetivo, en este artículo se caracteriza la fisionomía de la lectura en formatos digitales, especialmente en comparación con los impresos, utilizando datos del Estudio de Comportamiento Lector del año 2014 (Consejo Nacional de la Cultura y las Artes).

A continuación, se discute el rol de las tecnologías de la información en la lectura, así como los cambios ocurridos en la lectura como producto de los cambios tecnológicos. Luego se argumenta que las prácticas lectoras pueden ser mejor comprendidas como una práctica cultural y se discute la vinculación de éstas con determinados grupos sociales. Para dar sustento a esta discusión, se utilizan tres líneas teóricas pertinentes y posteriormente se describen los perfiles de distintos tipos de lectores en Chile según el uso de formatos digitales e impresos. Finalmente, se discute el rol de la educación de los padres en los perfiles encontrados y se problematizan los hallazgos a la luz del panorama actual del fenómeno lector en Chile.

\section{LA LECTURA Y LOS CAMBIOS TECNOLÓGICOS EN CHILE}

Los cambios tecnológicos apuntalados por la revolución digital de finales del siglo XX han transformado la cultura escrita y, con ello, las formas de leer. Una discusión ilustrativa al respecto es la que se instaura entre las posiciones definidas por Umberto Eco como apocalípticos e integrados. En síntesis, se trata de la oposición entre quienes veían la llegada de las nuevas tecnologías de la información al ámbito de la cultura con ojos catastróficos, frente a quienes percibían este cambio con especial optimismo. Por un lado, se auguraba la muerte del libro -al menos como había sido conocido hasta el momento-y, por otra parte, se defendían los rendimientos de aplicar estas tecnologías al mundo de la cultura escrita con particular optimismo. Para apocalípticos, los integrados despliegan un optimismo ingenuo en la medida en que le atribuyen un valor intrínseco a las nuevas tecnologías; para integrados, los apocalípticos poseen un espíritu de crítica pesimista y pusilánime, incapaz de distinguir las particularidades de las transformaciones tecnológicas en la cultura.

El tiempo transcurrido desde que Eco publicara su ensayo ha llevado a evaluaciones menos polarizadas. La tendencia seguida por la lectura de libros en dispositivos electrónicos es ilustrativa. Pese a un alza considerable de la lectura en formato digital (ebooks) en Estados Unidos (donde entre los años 
2011 y el 2014 el porcentaje de personas adultas que leen al menos un libro en formato digital al año se elevó de un 17 a un 28 por ciento), la lectura de libros en formato impreso se ha mantenido estable. Por ejemplo, la población mayor de dieciocho años que lee libros impresos representaba un 71 por ciento en 2011 y un 69 por ciento en 2014 (Pew Research Center). Fuera del área anglófona, la penetración de los libros digitales ha sido menos intensa y la venta de libros digitales se ha estancado en Europa y Brasil desde el año 2013 (Wischenbart). En el mercado del libro español, referente importante para Latinoamérica, el año 2011 los libros digitales representaron solamente alrededor de un 3 por ciento de la facturación editorial (Marcos). En el caso chileno, el año 2014 un 27 por ciento indicó haber leído algún libro digital, mientras que el 63,5 por ciento había leído un libro impreso (Consejo Nacional de la Cultura y las Artes, ver Tabla 1). De tal forma, sigue abierta la pregunta por los efectos que eventualmente tendrá el cambio tecnológico sobre las prácticas de lectura y las preferencias literarias.

Por otra parte, en Chile ha habido un importante avance en la disponibilidad de una de las principales tecnologías para acceder a materiales de lectura en formato digital, internet. En un lustro, el acceso a internet aumentó más del doble: mientras que el año 2009 se estimaba que un 30 por ciento de los hogares en Chile contaban con esta tecnología, al año 2014 se calculaba que dos tercios de los hogares en Chile contaban con conexión a internet ${ }^{3}$. No obstante, se constata una considerable desigualdad en el acceso según el quintil de ingresos del hogar, ya que se estima que el año 2016 un 54 por ciento de los hogares del quintil más pobre poseen internet frente al 86 por ciento del quintil más rico.

Además, la expansión de internet y las nuevas tecnologías a nivel global han modificado la producción literaria, dando paso, por ejemplo, a la incipiente literatura digital. Ésta se caracteriza por cobrar sentido en la materialidad digital, por ejemplo, a través de un marcado carácter hipertextual (Gainza). La literatura digital, por tanto, muestra que el formato le puede dar un carácter particular y distinto a ciertas producciones culturales. Asimismo, existe evidencia de que los materiales de lectura en formatos digitales e impresos tienen distintos usos. Tanto la lectura como operaciones simples de edición

El indicador del 2009 proviene de la Encuesta de Caracterización Socioeconómica de ese año, mientras que el del 2014 se estima a partir de la VI Encuesta Nacional de Acceso y Usos de Internet. 
de texto en formato impreso y digital muestran especificidades según el formato (Noyes y Garland). En otras palabras, no se debe considerar a los formatos digitales como una mera extensión de los impresos, sino que como poseedores de particularidades que deben ser estudiadas y caracterizadas.

\section{LA LECTURA COMO PRÁCTICA CULTURAL}

Las prácticas lectoras han vivido innegables cambios producto de las transformaciones tecnológicas recientes, generando importantes interrogantes acerca de los mecanismos que se encuentran en la base del gusto y la lectura. Para avanzar en la comprensión del fenómeno lector, argumentamos que la lectura debe ser entendida como una práctica cultural: el significado que produce la lectura para el actor y su entorno es inseparable del marco cultural en el cual ésta se inserta. Las prácticas lectoras consisten, por tanto, en una forma de participar en la cultura. A continuación, se exponen tres propuestas teóricas que permiten analizar la lectura como una práctica cultural: la teoría de la homología entre cultura y estructura social, la tesis del omnivorismo cultural, y la propuesta de Lahire sobre la consonancia de las prácticas culturales.

En primer lugar, existe un enfoque que destaca cómo las prácticas culturales, en general, se caracterizan por una realización restringida, segmentada por distintos criterios, como la educación o los recursos económicos de los individuos y sus hogares. La obra de Pierre Bourdieu, La distinción, constituye un hito fundante al respecto. En este trabajo se apunta a establecer las bases sociales del gusto, entendidas como los patrones que orientan las prácticas culturales -como la lectura- y las preferencias artísticas compartidas por individuos al interior de grupos o clases sociales. La propuesta presentada por el sociólogo francés se conoce como la tesis de la homología entre cultura y estructura social, en tanto se encontraría una correspondencia entre las prácticas culturales que se despliegan y el capital social, cultural y económico de los agentes. En otras palabras, quienes son parte de la elite tienen gustos y prácticas culturales exclusivas de este grupo, así como quienes provienen de sectores populares tienden a ser afines a prácticas y preferencias de la cultura popular.

Por otra parte, a comienzos de la década de 1990 surge en EE.UU. la tesis del omnivorismo que a partir de evidencia empírica plantea una apertura del abanico de prácticas y preferencias culturales entre los grupos más ricos (Peterson, "Understanding"; Peterson, "The rise"; Peterson y Kern). 
Es una interpretación que se ha considerado como contendora de la tesis de la homología entre cultura y estructura, en tanto encuentra un aumento de la tolerancia hacia prácticas culturales de distintos estatus, lo que minaría el patrón de exclusividad y correspondencia entre posición social y gustos propuesto por Bourdieu (Fernández Rodríguez y Heikkilä). En tal sentido, Peterson postula una distinción conceptual central entre los"omnívoros", quienes realizan prácticas con distintos estatus sociales, y los "unívoros", quienes poseen un abanico de prácticas reducido, ya sea a la alta cultura o a la cultura popular. En términos de las prácticas lectoras, desde esta corriente se propondría una disminución de las barreras entre tipos y formatos de lectura.

Una tercera propuesta teórica más reciente (Lahire) cuestiona la rigidez del modelo bourdieuano, así como las categorías del omnivorismo, y argumenta a favor de una comprensión más singularizada de las prácticas culturales. Para Bernard Lahire, en los individuos pueden coexistir prácticas potencialmente contradictorias en términos del estatus asociado a estas, es decir, pueden desplegar prácticas consideradas legítimas e ilegítimas simultáneamente. Por lo tanto, el autor plantea que existen individuos con un carácter plural, que no están determinados por patrones sociales dados, como por ejemplo el capital cultural. De esta forma, la distinción relevante que cabe hacer para Lahire es la de prácticas consonantes o disonantes, principalmente con la trayectoria personal. Mientras la tesis del omnivorismo apunta a un eclecticismo de las prácticas culturales entre grupos sociales, Lahire cambia el foco hacia un eclecticismo al interior del individuo.

Uno de los puntos de discusión entre estas perspectivas se encuentra en la interpretación de los hallazgos empíricos que muestran menos fragmentación entre las prácticas culturales que realiza la población en distintos países (Peterson, "Understanding"); es decir, en la observación de que las prácticas culturales se extienden por más grupos sociales que lo hallado por Bourdieu. Peterson ha considerado esto como un signo de erosión de las jerarquías culturales, mientras otros han dado cuenta de que este patrón corresponde más bien a una nueva forma de distinción (Coulangeon y Lemel; Fridman y Ollivier; Lizardo y Skiles). En esta última mirada se sugiere entonces que, al cruzar las fronteras establecidas previamente en la sociedad, los omnívoros trazan nuevas fronteras sociales, configurando una nueva fuente de distinción social (Hanquinet). Una ilustración de esto es la noción de "consumo irónico" que consiste en la realización de ciertas prácticas culturales que son consideradas como "malas" o inapropiadas en términos normativos, lo que produce una 
contradicción en la medida en que el consumo toma la forma de un "placer culpable" (McCoy y Scarborough).

\section{LAS PRÁCTICAS LECTORAS EN CHILE}

En el contexto chileno se han estudiado las prácticas culturales, en general (Gayo, Teitelboim y Méndez; Güell y Peters), y los libros y la lectura, en particular, desde distintos enfoques. Una perspectiva dominante se ha concentrado en caracterizar la participación de la población en las prácticas lectoras, en algunos casos considerando su desarrollo histórico (Cociña; Consejo Nacional de la Cultura y las Artes; Moya, "La lectura"; Subercaseaux; Torche). Entre los hallazgos de estos estudios, destaca la importancia que tiene el nivel educacional de las personas y de los padres sobre la lectura de libros, así como los ingresos del hogar (Cociña). También se ha relevado el rol de la disposición hacia la lectura, específicamente del gusto por la lectura en general, como un factor determinante del leer libros, más allá del nivel educacional (Moya, "La lectura").

Según Torche, la lectura de libros constituye un marcador de estatus en Chile, a diferencia de otros materiales como los periódicos y las revistas. En esta línea, cabe destacar que la lectura está supeditada a ciertas valoraciones sociales (Donnat; Moya, "Los aportes"); para algunos puede ser una práctica noble y edificante, mientras que para otros una cuestión aburrida e incluso tortuosa. Por lo mismo, al interior de una sociedad dada se constituyen desigualdades en torno a las prácticas lectoras y a la valoración asociada a éstas. Por ejemplo, en la actualidad las lecturas literarias -como la narrativa y la poesía- suelen ser más valoradas por la sociedad que las de carácter funcional -como el leer correos electrónicos o folletos-.

El corolario de la evidencia respecto a las prácticas lectoras en Chile, especialmente la referida a los libros, es que éstas siguen patrones de restricción según características sociales, tales como el nivel educacional y los recursos económicos. Esto respalda una interpretación en la línea de la homología entre prácticas culturales y estructura social presentada. Sin embargo, también se ha planteado que existen limitantes para la hipótesis de la homología en el caso chileno. Si bien existen patrones sociales de fragmentación y exclusividad de las prácticas culturales (Gayo, Teitelboim y Méndez), también se encuentran indicios de prácticas orientadas más individualmente (Güell y Peters). Güell, 
Peters y Morales plantean que "el uso de las tecnologías como instancias mediadoras del consumo cultural, ha configurado un tipo de consumidor que accede a un sinfín de nuevos bienes y servicios culturales donde la presencia no es un factor determinante" (104), por lo que cabe analizar qué cambios pueden encontrarse en las lecturas en formatos digitales, atendiendo las eventuales transformaciones que la introducción de una innovación de formato puede tener en estas prácticas culturales.

Si bien existe bastante acuerdo entre los autores en considerar las prácticas lectoras como una práctica cultural fuertemente vinculada al capital social, cultural y económico de las personas, ha habido poca atención a formatos distintos al libro, dejando fuera otras prácticas lectoras cotidianas y omnipresentes en la sociedad, como la lectura de periódicos, revistas e historietas. Además, se han desatendido las prácticas de lectura en formatos digitales, siendo uno de los aspectos que no ha sido debidamente desarrollado y problematizado, así como la relación que se evidencia entre la lectura en estos formatos con los impresos. En esta dirección, la pregunta que guía el presente artículo se refiere a cuál es la fisionomía de la lectura en formatos digitales, especialmente en comparación con los impresos. Una cuestión central será evaluar el perfil de los grupos de lectores en formatos digitales e impresos, para revisar si se sostienen las viejas desigualdades sociales en torno a los formatos impresos. Para esto, se plantea una segmentación de los lectores según sus prácticas lectoras declaradas de libros, revistas, periódicos e historietas, en impresos y digitales.

\section{LECTURA EN FORMATOS DIGITALES EN CHILE}

Al investigar la lectura, esta muchas veces ha sido tratada como una operación abstracta y genérica, donde un individuo interpreta un texto de manera silenciosa e introspectiva. Indistintamente del género y formato que se lea, esta perspectiva supone que existe una operación universal en la lectura (Hillesund). Sin embargo, existe abundante evidencia respecto a las diferencias producidas en la lectura en escenarios específicos, según varíe el tipo de texto, el formato en que se presenta y el entorno social en que se inserta (Cavallo y Chartier; Darnton; Manguel; Noyes y Garland). Una variante importante, y en la que nos centraremos en este artículo, es la diferencia que se observa en la lectura en formatos digitales e impresos para el caso chileno en la actualidad. 
Dentro de las especificidades de la lectura en formato digital, particularmente la que se realiza en internet, un estudio en Argentina da cuenta de que ésta aparece como una "lectura navegación", que "adquiere una dimensión interactiva que no existía en los dispositivos anteriores" (Albarello 167). El estudio también destaca que el formato digital -la lectura en pantalla-y el formato impreso corresponden a dos modos diferentes de lectura (Eden y Eshet-Alkalai; Hillesund; Noyes y Garland; Peronard). El estudio de Albarello establece, además, que la lectura digital destaca por ocurrir en un contexto de multitareas, donde se realizan simultáneamente distintas actividades. Destaca, a su vez, que "la pantalla y el impreso ofrecen distintas experiencias de placer durante el acto de lectura" (208) y que, en ese sentido, se exhiben diferencias generacionales entre lectores nacidos antes o después de 1980 . Estas diferencias generacionales se explican a partir de una mayor o menor familiaridad con las tecnologías digitales.

Para hacer una caracterización de la lectura en formatos digitales en Chile se utilizarán los datos del Estudio de Comportamiento Lector 2014, encuesta realizada por el Consejo Nacional de la Cultura y las Artes. Este estudio considera una muestra probabilística estratificada geográficamente y polietápica de 6.990 personas. Los datos son representativos de hombres y mujeres de 9 a 65 años, residentes en zonas urbanas de las 15 regiones de Chile. La población de referencia utilizada aquí y en el resto del texto se acota a la edad de 15 a 65 años ${ }^{4}$.

La Tabla 1 muestra la distribución de las prácticas lectoras en Chile el año 2014 para cuatro materiales de lectura en formato digital e impreso ${ }^{5}$. Estos materiales corresponden a libros, revistas, periódicos e historietas. Un primer resultado de interés es la tendencia general a una menor lectura de formatos digitales en comparación con los impresos. La proporción de lectores en formato impreso se reduce aproximadamente a la mitad para cada material de lectura en formato digital.

$4 \quad$ Se decidió elevar la edad mínima a ser considerada, en tanto se busca caracterizar el grupo de la población que tiene mayor autonomía sobre sus prácticas lectoras.

Las preguntas tienen la forma "En los últimos 12 meses, ¿ha leído [material de lectura] en formato [impreso/digital]?", haciendo variar el material de lectura y el formato para cada uno. 
Tabla 1. Lectura de cuatro materiales al menos una vez en los últimos doce meses en formato digital e impreso (porcentaje)

\begin{tabular}{lll}
\hline & Digital & Impreso \\
\hline Libros & 27,0 & 63,5 \\
Revistas & 18,0 & 52,8 \\
Periódicos & 38,3 & 74,8 \\
Historietas & 9,5 & 24,7 \\
\hline
\end{tabular}

Fuente: Elaboración propia a partir de Estudio de Comportamiento Lector 2014

Entre los cuatro materiales de lectura son los periódicos impresos los más extendidos, pues tres cuartos de la población ha leído alguno en los últimos doce meses. Le siguen los libros y las revistas impresas. El caso de las historietas o cómics es el menos común, ya que alrededor de un décimo de la población las lee en formato digital y un cuarto en impreso.

Estos resultados evidencian una tendencia similar a la de otros países respecto a las prácticas de lectura en formatos digitales e impresos. Los formatos digitales están menos extendidos entre las prácticas lectoras de la población en comparación con los impresos en estos cuatro materiales de lectura.

Ahora bien, para profundizar en una caracterización del lugar que ocupan las lecturas en formatos digitales en la fisionomía actual de las prácticas lectoras en Chile, cabe caracterizar a las personas según si leen o no en formatos digitales e impresos. Particularmente, para cada material de lectura se construyen grupos de quienes no leen ninguno de los formatos, de quienes leen exclusivamente el formato impreso y no el digital, de quienes leen exclusivamente el formato digital y no el impreso, y de quienes leen el material en ambos formatos. Asumiendo que los formatos digitales no son una mera duplicación de los impresos, es posible considerar al grupo de personas que lee ambos formatos como el grupo con las prácticas lectoras más diversas. En este sentido, se espera que los lectores de ambos formatos sigan un patrón más bien omnívoro en lo que respecta a sus prácticas lectoras. Aquellos que solo leen en formato digital probablemente son personas más bien jóvenes, cuyas prácticas culturales, en general, están mediadas por las nuevas tecnologías en el sentido propuesto por Güell y Peters. Por otra parte, aquellos que solo leen en formato impreso o que no leen los materiales son 
grupos que han recibido más atención en la literatura y sirven especialmente como punto de comparación. Para caracterizar estos grupos se utilizan variables sociodemográficas: sexo, edad, nivel educacional e ingresos del hogar, así como ítems que miden el gusto por la lectura en general ${ }^{6} \mathrm{y}$ el número de horas promedio en que se usa internet los siete días de la semana (ver Tabla 2).

Tabla 2. Caracterización de lectores según materiales y grupo de lectores

\begin{tabular}{|c|c|c|c|c|}
\hline & Ninguno & Solo impreso & Solo digital & Ambos \\
\hline \multicolumn{5}{|l|}{ Libros } \\
\hline Porcentaje & 29,6 & 43,6 & 3,4 & 23,4 \\
\hline Edad (media) & 41,3 & 39,8 & 31,3 & 31,0 \\
\hline Mujer (porcentaje) & 43,8 & 57,8 & 39,7 & 49,6 \\
\hline \multicolumn{5}{|l|}{ Educación (porcentaje) } \\
\hline Media incompleta o menos & 47,0 & 33,2 & 18,0 & 21,7 \\
\hline Media completa & 34,5 & 33,9 & 26,2 & 18,7 \\
\hline Superior & 18,5 & 32,9 & 55,8 & 59,6 \\
\hline Horas internet semana (media) & 3,93 & 5,13 & 10,27 & 9,49 \\
\hline Gusto por la lectura (media) & 2,78 & 3,77 & 3,61 & 4,07 \\
\hline Ingresos hogar en pesos (media) & 584.383 & 748.297 & 919.369 & 974.064 \\
\hline \multicolumn{5}{|l|}{ Revistas } \\
\hline Porcentaje & 44,9 & 38,2 & 3,2 & 13,7 \\
\hline Edad (media) & 38,3 & 40,3 & 30,1 & 32,3 \\
\hline Mujer (porcentaje) & 45,2 & 59,4 & 41 & 46,2 \\
\hline \multicolumn{5}{|l|}{ Educación (porcentaje) } \\
\hline Media incompleta o menos & 40,2 & 35,7 & 12,9 & 19 \\
\hline Media completa & 31,5 & 34,5 & 25,6 & 16,8 \\
\hline Superior & 28,4 & 29,7 & 61,5 & 64,3 \\
\hline Horas internet semana (media) & 4,95 & 5,05 & 9,86 & 10,15 \\
\hline Gusto por la lectura (media) & 3,13 & 3,70 & 3,80 & 4,08 \\
\hline Ingresos hogar en pesos (media) & 661.587 & 742.005 & 931.567 & 1.022 .184 \\
\hline
\end{tabular}

6 Medido a través de la pregunta “En general, ¿cuánto le gusta leer?”, con cinco categorías que van de "nada" a "me gusta mucho". 


\begin{tabular}{lllll}
\hline Periódicos & & & & \\
Porcentaje & 21,3 & 43,1 & 7,7 & 27,8 \\
Edad (media) & 35,8 & 42,2 & 31,6 & 34,4 \\
Mujer (porcentaje) & 55,3 & 53,4 & 41,7 & 46,3 \\
Educación (porcentaje) & & & & \\
$\quad$ Media incompleta o menos & 53,3 & 43,4 & 17,4 & 15,2 \\
$\quad$ Media completa & 23,5 & 36,5 & 26,9 & 25,8 \\
$\quad$ Superior & 23,3 & 20,1 & 55,7 & 59,1 \\
Hora internet semana (media) & 5,06 & 3,54 & 10,06 & 8,81 \\
Gusto por la lectura (media) & 2,95 & 3,44 & 3,55 & 3,93 \\
Ingresos hogar en pesos (media) & 676.863 & 599.774 & 923.265 & 978.814 \\
\hline Historietas & & & & \\
Porcentaje & 68,6 & 21,4 & 3,5 & 6,5 \\
Edad & 39,0 & 38,2 & 28,6 & 29,8 \\
Mujer (porcentaje) & 52,9 & 48,8 & 40,7 & 34,9 \\
Educación (porcentaje) & & & & \\
$\quad$ Media incompleta o menos & 34,2 & 41,0 & 22,5 & 23,5 \\
$\quad$ Media completa & 30,7 & 33,7 & 21,6 & 20,3 \\
$\quad$ Superior & 35,1 & 25,3 & 55,9 & 56,2 \\
Hora internet semana (media) & 5,65 & 4,87 & 9,90 & 10,48 \\
Gusto por la lectura (media) & 3,39 & 3,72 & 3,91 & 4,11 \\
Ingresos hogar en pesos (media) & 746.105 & 701.283 & 933.228 & 815.339 \\
\hline
\end{tabular}

Fuente: Elaboración propia a partir de Estudio de Comportamiento Lector 2014

La Tabla 2 muestra la comparación de las características de los lectores de los cuatro materiales de lectura considerados, en formato digital e impreso. En el caso de los libros, la mayoría de los encuestados $(43,6 \%)$ reportó leer solamente libros impresos, seguido de aquellos encuestados que no leen libros en ningún formato $(29,6 \%)$ y de los que leen ambos formatos $(23,4 \%)$. Una minoría de los encuestados reportó leer solamente en formato digital $(3,4 \%)$. Esta última tendencia se repite en todos los materiales de lectura: quienes leen exclusivamente formatos digitales son una proporción muy baja de los encuestados, el caso donde representan una mayor proporción es en los periódicos $(7,7 \%)$. En el caso de las revistas, la mayor parte no las lee en ningún formato $(44,9 \%)$ o bien en formato impreso $(38,2 \%)$. Quienes reportan leer revistas en ambos formatos constituyen solamente alrededor de 
un octavo de los encuestados. Los periódicos son el material de lectura que tiene la mayor proporción de lectores omnívoros, en tanto más de un cuarto de los encuestados reporta leerlos en ambos formatos. Por último, el caso de las historietas o cómics es el que presenta la menor proporción de lectores que usan ambos formatos $(6,5 \%)$.

Los resultados exhiben importantes diferencias en la composición social de los perfiles de lectores de libros: son las personas con mayores ingresos y nivel educacional las que presentan las prácticas más diversas de lectura según formatos, leyendo tanto libros impresos como digitales, así como el mayor gusto promedio por la lectura. Por ejemplo, mientras los omnívoros de libros tienen ingresos del hogar promedio que superan los 950 mil pesos, los lectores de libros impresos reciben ingresos promedio menores a 750 mil pesos y los que no leen tienen ingresos promedio menores a 600 mil pesos. Lo mismo ocurre con el nivel educacional: el 59,6 por ciento de las personas que lee libros tanto impresos como digitales tiene educación superior, mientras que solamente el 32,9 y el 18,5 por ciento de los que leen impresos o no leen, se encuentran en la misma situación. El caso de los lectores de libros digitales es un tanto particular: si bien tienen niveles de ingreso y educación similares a los de los omnívoros, tienden a ser en mayor medida hombres $(60,3 \%)$ y a usar internet por un número superior de horas promedio a la semana que el resto de los grupos (10,27 horas). Finalmente, cabe destacar que la edad promedio es menor para el grupo de lectores omnívoros (31,3 años) y de quienes solo leen en formato digital (31 años) que para el grupo de los que no leen libros (41,3 años) o los que solo los leen formato impreso (39,8 años).

La composición de los lectores de revistas agrupados según formatos sigue un patrón similar al de los libros. Los omnívoros, es decir, quienes leen ambos formatos, son los que han cursado educación superior en mayor proporción $(64,3 \%)$, mientras que menos de un tercio $(28,3 \%)$ de quienes leen libros exclusivamente en formato impreso poseen este nivel educacional. Además, los omnívoros son quienes tienen mayores ingresos promedio del hogar y, a su vez, manifiestan en promedio un mayor gusto por la lectura y un mayor uso de internet a la semana en comparación al grupo de los que leen solo en formato impreso y quienes no leen libros. Quienes leen revistas solo en formato digital tienen un perfil muy similar al de los omnívoros, como ocurría en el caso de los libros.

El caso de los periódicos también muestra una diferencia importante según los tipos de formatos que se lee, en un sentido similar al de los libros y las revistas. Quienes leen periódicos en formatos digitales e impresos 
han cursado en mayor medida educación superior y tienen en promedio el mayor gusto por la lectura. La diferencia entre la edad promedio del grupo de omnívoros con el no de lectores es menor, no obstante, quienes solo leen periódicos en formatos impresos tienden a ser mayores que el resto de los grupos en promedio.

Las historietas y cómics siguen la misma tendencia descrita en la comparación entre el grupo de los omnívoros y los que solo leen en formato impreso o no leen historietas. La excepción que destaca es que la composición por género del grupo de los omnívoros es más bien masculina, pues las mujeres constituyen una proporción menor de estos lectores (34,9\%).

En definitiva, se observa que existen importantes diferencias en las características de quienes son lectores omnívoros en términos de los formatos digitales e impresos para los cuatro materiales analizados. El perfil de quienes solo leen en formato digital tiende a parecerse al de los omnívoros, no obstante representa un grupo muy reducido en términos proporcionales. Los resultados constituyen evidencia respecto a que quienes tienen prácticas omnívoras corresponden a grupos más aventajados socialmente, con mayor educación e ingresos del hogar.

\section{CONTINUIDADES Y RUPTURAS ENTRE LO IMPRESO Y LO DIGITAL}

La fisionomía de las lecturas en formatos digitales e impresos da cuenta de que quienes tienen las prácticas más diversas en formatos corresponden a grupos aventajados socialmente, con una mayor disposición hacia la lectura de libros. Han desarrollado un gusto que los lleva a buscar materiales de lectura tanto en los tradicionales impresos como en las pantallas de distintos dispositivos electrónicos. En contraste, quienes solo leen formatos impresos son quienes tienen menos educación y un menor gusto por la lectura en relación con los omnívoros. Cabe profundizar respecto a la relación de este hallazgo sobre la población residente en Chile para el año 2014 en un sentido histórico.

La tesis de la homología plantea que la correspondencia entre posiciones sociales y prácticas culturales que se observa en un punto del tiempo también tiene un correlato histórico. Es decir, la explicación de este ordenamiento del gusto según patrones sociales no es espontánea, sino histórica. Para evaluar el sentido histórico que tiene la desigualdad en los perfiles sociales de los 
lectores en formatos digitales e impresos se analiza el nivel educacional de los padres y las madres de los encuestados ${ }^{7}$. Se restringe el análisis al material libro, considerando su estatus particular y dado que los resultados son consistentes con el resto de los materiales.

Las mismas diferencias en la composición de los perfiles de lectores pueden observarse si se analizan los niveles educacionales de las madres y los padres de los encuestados. En la Tabla 3 se evidencia que el 45,7 por ciento de los lectores que leen libros, tanto digitales como impresos, tiene al menos un padre con educación superior, frente al 22,8 por ciento de quienes solamente leen libros impresos y al 11,1 por ciento de los que no leen libros. Es decir, se observa una importante continuidad generacional en el efecto de la educación en la tendencia a presentar prácticas lectoras en formatos más diversos.

En definitiva, en la relación entre los formatos impresos y digitales se constatan continuidades y rupturas. Por una parte, existen rupturas propias de la época en las prácticas lectoras, dadas por la introducción de nuevas tecnologías. El formato digital genera una nueva distinción dentro de la lectura, en tanto se diferencian los lectores que solo leen en formato impreso de los que leen en ambos formatos o solo en digital. Por otra parte, se constata una continuidad entre los formatos impresos y digitales en términos de la reproducción intergeneracional de la educación sobre la desigual disposición a leer libros en distintos formatos.

Tabla 3. Mayor nivel educacional alcanzado por la madre o el padre de grupos de lectores de libros (porcentaje)

\begin{tabular}{lllll}
\hline & Ninguno & Solo impreso & Solo digital & Ambos \\
\hline Básica completa o menos & 53,9 & 39,4 & 16,8 & 18,4 \\
Media completa & 35,0 & 37,9 & 42,6 & 35,9 \\
Superior & 11,1 & 22,8 & 40,6 & 45,7 \\
\hline
\end{tabular}

Fuente: Elaboración propia a partir de Estudio de Comportamiento Lector 2014 


\section{DISCUSIÓN Y CONCLUSIONES}

Las prácticas lectoras son un fenómeno social complejo que requiere atender distintas aristas, tanto en la realización de las mismas como en sus valoraciones. En particular, los perfiles de lectores construidos según los formatos digitales e impresos que son leídos dan cuenta de desigualdades sociales relevantes que permiten avanzar en la comprensión del fenómeno lector en el contexto chileno actual.

La introducción de nuevas tecnologías en la cultura escrita trae consigo cambios en los materiales y en las prácticas de lectura. En Chile, la lectura de libros, revistas, periódicos e historietas en formato digital todavía es incipiente en comparación a sus pares impresos. Sin embargo, existen proporciones considerables de la población que leen los formatos digitales. La tendencia general indica que los lectores de estos formatos tienden a combinar prácticas en formatos impresos y digitales. Se configuran como un perfil de lector omnívoro, en tanto presentan un acercamiento más diverso a los formatos de lectura. Por su parte, quienes leen exclusivamente en digital son todavía una proporción muy menor de la población, caracterizada por tener un perfil social similar al de los omnívoros.

Siguiendo las directrices teóricas sobre las prácticas culturales y la participación cultural, los resultados apuntan a una interpretación de las prácticas lectoras en formato digital en la línea de la homología entre cultura y estructura social, en tanto se observan patrones sociales marcados por el nivel educacional y los ingresos del hogar. No obstante, como la lectura en este formato tiende a coexistir con el impreso, cabe preguntarse por el sentido que tiene esta aproximación omnívora. Según lo analizado, este eclecticismo hacia los formatos antes que deberse a una propensión individual, constituye más bien un patrón social dado por el nivel educacional y los ingresos del hogar.

Por lo tanto, los resultados siguen solo parcialmente la tesis de la homología. El perfil omnívoro en este caso sugiere una forma de trazar barreras simbólicas, en tanto se constituye como un grupo aventajado socialmente, con un perfil similar al de los que exclusivamente leen en formatos digitales. Esto sigue el patrón encontrado por otros estudios donde se asocia el omnivorismo a una nueva estrategia de distinción (Coulangeon y Lemel) y a un discurso ostentatorio de la diversidad donde se vincula el eclecticismo de las prácticas culturales con una visión más abierta y tolerante, propia de grupos privilegiados (Fridman y Ollivier). En este sentido, la mayor disposición de los omnívoros hacia 
materiales de lectura de distinto estatus, expresada como un mayor promedio en el gusto por la lectura en general, es un hallazgo que parece situarse en la línea del eclecticismo y la demarcación de nuevas fronteras de distinción.

Otro hallazgo destacable se refiere a la asociación entre la lectura en formatos digitales y una menor edad promedio para todos los materiales de lectura en comparación con los impresos. Siguiendo el umbral propuesto por Albarello, en términos de la familiaridad con la tecnología de quienes nacieron desde 1980 en adelante, el promedio de edad de los grupos de lectores en ambos formatos o exclusivamente digital está en general por debajo de los 34 años (edad que tendría para el año de la encuesta una persona nacida en 1980). Los formatos digitales son una innovación reciente en la cultura escrita, lo que se condice con que la población más afín a este formato tiende a ser más joven.

$\mathrm{Al}$ analizar el nivel educacional de los padres, se reproduce la diferencia encontrada en la educación de los grupos de lectores. Aquello da pie para plantear una reproducción intergeneracional de la desigual disposición hacia la lectura, en tanto quienes han tenido padres con mayor educación tienden a desarrollar un mayor gusto por la lectura y, por tanto, una disposición hacia la lectura de distintos materiales de lectura en distintos formatos. En este sentido, se constata una persistencia de viejas desigualdades sociales en la participación de la población en Chile en la lectura de libros y otros materiales.

Las nuevas prácticas lectoras en formatos digitales ciertamente responden a un cambio de época donde emergen nuevas formas de leer gracias a las nuevas tecnologías. Una ilustración de esto lo constituyen aquellos textos que hacen un uso extensivo de características como el hipertexto, requiriendo nuevas estrategias de lectura. No obstante, a partir de los resultados presentados aquí,también se puede reconocer en la lectura de formatos digitales una importante y desafiante continuidad en las desigualdades sociales que han caracterizado las prácticas lectoras en formato impreso. Se trata de una continuidad importante en la medida en que las prácticas de lectura atraen la atención de distintos actores, incluido el mundo intelectual, que buscan extenderlas en la población. A la vez, esta continuidad se torna desafiante en tanto los ingentes esfuerzos que se dedican a la expansión de la cultura escrita y a la superación de las viejas desigualdades sociales que atraviesan la lectura ahora tienen que ampliarse hacia los formatos digitales, propios de la nueva época inaugurada con la incorporación de las nuevas tecnologías. 


\section{BIBLIOGRAFÍA}

Albarello, Francisco. Leer/navegar en Internet: las formas de lectura en la computadora. Buenos Aires: La Crujía, 2011.

Bourdieu, Pierre. La distinción: criterio y bases sociales del gusto. Madrid: Taurus, 2012. Print.

Cavallo, Guglielmo y Roger Chartier. Historia de la lectura en el mundo occidental. Madrid: Taurus, 2004.

Cociña, Matías. "Determinantes de la lectura en Chile". Tesis para optar al grado de Magíster en Economía Aplicada. Universidad de Chile, 2007.

Consejo Nacional de la Cultura y las Artes. Estudio de Comportamiento Lector 2014 [Archivo de datos]. 2014. Web. 15 jul. 2016.

Consejo Nacional de la Cultura y las Artes. Prácticas de consumo, participación y valoración de la cultura en Chile: etnografía de análisis de casos. Santiago de Chile: CNCA, 2014.

Coulangeon, Philippe y Yannick Lemel. "Social status and cultural consumption in seven countriesIs 'distinction' really outdated? Questioning the meaning of the omnivorization of musical taste in contemporary France”. Poetics 35.2 (2007): 93-111. ScienceDirect.

Darnton, Robert. "Historia de la lectura". Formas de hacer historia. Ed. Peter Burke. Madrid: Alianza, 1996.

Donnat, Olivier. "Encuestas sobre los comportamientos de lectura. Cuestiones de método". Sociología de la Lectura. Comp. Bernard Lahire.Barcelona: Gedisa, 2005.

Eco, Umberto. Apocalípticos e integrados. España: Lumen, 1984.

Eden, Sigal y Yoram Eshet-Alkalai. "The Effect of Format on Performance: Editing Text in Print versus Digital Formats". British Journal of Educational Technology 44.5 (2013): 846-856. Wiley Online Library.

Fernández Rodríguez, Carlos Jesús y Riie Heikkilä. "El debate sobre el omnivorismo cultural. Una aproximación a nuevas tendencias en Sociología del Consumo". Revista internacional de sociología 69.3 (2011): 585-606.

Fridman, Viviana y Michèle Ollivier. "Ouverture ostentatoire à la diversité et cosmopolitisme: Vers une nouvelle configuration discursive ?" Sociologie et sociétés 36.1 (2004): 105. CrossRef.

Gainza, Carolina. "Experiencias hipertextuales: escritura y lectura en digital". Un lugar para los libros. Reflexiones del encuentro nacional de cultura escrita y prácticas lectoras. Coord. Cristóbal Moya y Lorena Fuentes. Santiago de Chile: Lom, 2016.

Gayo, Modesto, Berta Teitelboim y María Luisa Méndez. "Exclusividad y fragmentación: Los perfiles culturales de la clase media en Chile". Universum: revista de humanidades $y$ ciencias sociales 28.1 (2013): 97-128.

Güell, Pedro y Tomás Peters. La trama social de las prácticas culturales en Chile: Sociedady subjetividad en el consumo cultural de los chilenos. Santiago de Chile: Ediciones UAH, 2012.

Güell, Pedro, Tomás Peters y Rommy Morales. "Tipología de prácticas de consumo cultural en Chile a inicios del siglo XXI: mismas desigualdades, prácticas emergentes, nuevos desafíos". La trama social de las prácticas culturales en Chile: Sociedad y subjetividad en el consumo cultural de los chilenos. Santiago de Chile: Ediciones UAH, 2012. 
Hanquinet, Laurie. "Exploring Dissonance and Omnivorousness: Another Look into the Rise of Eclecticism". Cultural Sociology (2016): doi: 10.1177/1749975516647750. cus. sagepub.com.

Hillesund, Terje. "Digital Reading Spaces: How Expert Readers Handle Books, the Web and Electronic Paper”. First Monday 15.4 (2010): uncommonculture.org. Web. 2 jul. 2016.

Lahire, Bernard. La culture des individus: dissonances culturelles et distinction de soi. Paris: La Découverte, 2006.

Lizardo, Omar y Sara Skiles. "Cultural Consumption in the Fine and Popular Arts Realms". Sociology Compass 2.2 (2008): 485-502.

Manguel, Alberto. Una historia de la lectura. Buenos Aires: Siglo XXI, 2014.

Marcos, Ana. “El ‘ebook' despega... pero vuela bajo”. El País 13 jul. 2012. Web. 13 jul. 2016.

McCoy, Charles Allan y Roscoe C. Scarborough. "Watching 'bad' television: Ironic consumption, camp, and guilty pleasures". Poetics 47 (2014): 41-59.

Moya, Cristóbal. "La lectura de libros en Chile: una práctica cultural dispuesta por el gusto". Tesis para optar al grado de Magíster en Ciencias Sociales mención Sociología. Universidad de Chile, 2013.

"Los aportes de la sociología para una agenda de investigación sobre prácticas lectoras en Chile". Un lugar para los libros. Reflexiones del encuentro nacional de cultura escrita y prácticas lectoras. Coord. Cristóbal Moya y Lorena Fuentes. Santiago de Chile: Lom, 2016.

Noyes, Jan M. y Kate J. Garland. “Computer- vs. Paper-Based Tasks: Are They Equivalent?" Ergonomics 51.9 (2008): 1352-1375.

Peronard, Marianne. "Lectura en papel y en pantalla de computador". Revista signos 40.63 (2007): 179-195.

Peterson, Richard A. "The rise and fall of highbrow snobbery as a status marker". Poetics 25.2 (1997): 75-92.

"Understanding audience segmentation: From elite and mass to omnivore and univore". Poetics 21.4 (1992): 243-258.

Peterson, Richard A. y Roger M. Kern. "Changing Highbrow Taste: From Snob to Omnivore". American Sociological Review 61.5 (1996): 900.

Pew Research Center. E-Reading rises as Device Ownership Jumps. 2014. Disponible en: http://pewinternet.org/Reports/2014/E-Reading-Update.aspx

Subercaseaux, Bernardo. Historia del libro en Chile. Santiago de Chile: Lom, 2010.

Torche, Florencia. "Social status and cultural consumption: The case of reading in Chile". Poetics 35.2-3 (2007): 70-92.

Wischenbart, Rüdiger. Global eBook 2016. Viena: RWCC, 2016. 